\title{
CONF- $9605194--2$
}

\section{ERNEST DRLANDU LAWRENCE BERKELEY NATIONAL LABDRATDRY}

\section{Intriguing Aspects of Strangeness Production at CERN Energies}

G. Odyniec

Nuclear Science Division

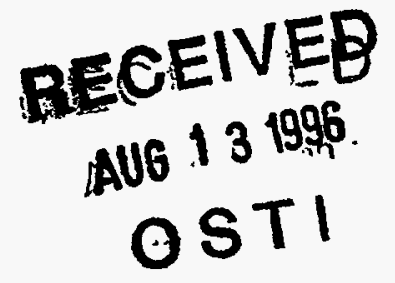

July 1996

To be presented at the Strangeness ' 96 Conference, Budapest, Hungary, May 15-17, 1996, and to be published in the Proceedings 


\section{DISCLAIMER}

This document was prepared as an account of work sponsored by the United States Government. While this document is believed to contain correct information, neither the United States Government nor any agency thereof, nor The Regents of the University of California, nor any of their employees, makes any warranty, express or implied, or assumes any legal responsibility for the accuracy, completeness, or usefuiness of any information, apparatus, product, or process disclosed, or represents that its use would not infringe privately owned rights. Reference herein to any specific commercial product, process, or service by its trade name, trademark, manufacturer, or otherwise, does not necessarily constitute or imply its endorsement, recommendation, or favoring by the United States Government or any agency thereof, or The Regents of the University of Califomia. The views and opinions of authors expressed herein do not necessarily state or reflect those of the United States Government or any agency thereof, or The Regents of the University of California. 
LBNL-39043

\title{
Intriguing Aspects of Strangeness Production at CERN Energies
}

Invited paper presented at the Strangeness ' 96 Conference, Budapest, Hungary, May 15-17, 1996

\author{
Grazyna Odyniec \\ Nuclear Science Division, Lawrence Berkeley National Laboratory \\ University of California, Berkeley, CA 94720
}

July 1996

This work was supported by the Director, Office of Energy Research, Division of Nuclear Physics of the office of High Energy and Nuclear Physics of the U.S. Department of Energy under Contract DE-AC03-76SF00098 


\section{DISCLAIMER}

Portions of this document may be illegible in electronic image products. Images are produced from the best available original document. 


\title{
Intriguing Aspects of Strangeness Production at CERN Energies.
}

\author{
Grażyna J. Odyniec \\ Lawrence Berkeley National Laboratory, \\ Berkeley, CA 94720, USA.
}

Abstract. Strange particle production in $\mathrm{pp}, \mathrm{pA}$ and $\mathrm{AA}$ collisions at CERN SPS energies is reviewed. First results from $\mathrm{Pb}$ beam experiments are briefly presented. The emerging picture (still incomplete) is discussed.

\section{Introduction}

Since the first report [1] of strangeness enhancement in heavy ion collisions at CERN SPS energies, strangeness became one of the most discussed and speculated on candidates for messenger of the new phase of the nuclear matter, Quark Gluon Plasma (QGP). Vast amounts of experimental data and theoretical calculations led to the series of conferences dedicated specifically to the strangeness, with the first one in Tucson last year, and the second one here, in Budapest.

Strangeness enhancement as a consequence of the formation of plasma phase was predicted already more than a decade ago [2]. It was argued that the high parton density and the lower energy threshold for $s \bar{s}$ pair production in the quark gluon plasma in comparison to the hadron gas lead to an increase of the strangeness content in the final state. These expectations were soon confirmed by lattice QCD calculations [3].

\section{Experimental approach and choice of the observables}

The most natural question is: How would we define and measure strangeness enhancement? The most commonly used definition is related to the comparison with nucleus-nucleus data; it says that strangeness enhancement observed in high energy nuclear collisions is understood as an increase of the total yield of $s \bar{s}$ quark pairs relative to the total yield of non-strange quark-antiquark pairs when comparing central nucleus-nucleus (A-A) collisions and nucleon-nucleon $(N-N)$ interactions at the same collision energy per nucleon. It is often expressed quantitatively, by the ratio of strangeness suppression factors $\lambda_{s}$, commonly used in high energy physics [4].

Relativistic heavy ion collisions provide a unique opportunity to create an environment (high energy density over large volumes) in which formation of the quark 
gluon plasma can be observed. Since typical temperatures of QGP are expected to be quite low (about $150-250 \mathrm{MeV}$ ), it is essential to be able to observe strange hadrons at low $p_{t}$ as they are expected to emerge from the plasma. It is very important to study total strange particle yields in all of phase space, because the relative particle yields in restricted phase space regions are known to be affected by kinematical effects, which significantly complicate interpretation.

Integrated yields of strange particles have several special properties, which make them very attractive to study. They have relatively high cross sections (easy to measure), they are independent of assumptions concerning system expansion and details of hadronization and freeze-out conditions (integrated over final state), and they are correlated only by the collision dynamics. However, they contain less detailed information than spectra.

We will start discussion of strangeness enhancement from systematic comparison of integrated yields in full phase space in $\mathrm{pp}, \mathrm{pA}$ and $\mathrm{AA}$ collisions. Later we will proceed to analysis of spectra.

\section{Strangeness in $\mathrm{pp}, \mathrm{pA}$, and SA collisions}

\subsection{Integrated yields}

Let us start from a not so encouraging statement that ... experiments are difficult (!), and there are almost no parameters to measure directly.

Therefore, for physics interpretation one often needs to rely on theoretical models. However, integrated yields are the only way to define strangeness enhancement independently of kinematic cuts, choice of variables and models (almost!, note that even in the analysis of $\mathrm{pA}$ collisions in "full" phase space, one needs to make assumptions regarding target fragments) which allow for comparison between systems, energies, etc.

All data presented in this chapter cover the full phase space. Particle yields for $\mathrm{pp}$ and $\mathrm{pA}$ at $200 \mathrm{GeV} / \mathrm{c}$ were obtained with visual tracking detectors (bubble chambers and streamer chambers) and therefore, they have rather large statistical errors, but low systematic uncertainties.

Fig.1 a,b and Fig.2 a,b show the ratios of the average $\Lambda$ (a) and $K_{s}^{\circ}(\mathrm{b}) \mathrm{mul}-$ tiplicities to the average multiplicity of negative hadrons, $\left\langle h^{-}\right\rangle$, in proton-nucleon (open circle) and proton-nucleus (full circles, Fig. 1) and $S+S$ (full circles, Fig. 2) interactions as a function of $\left\langle h^{-}\right\rangle$at $200 \mathrm{GeV}$. The full square in Fig.2b indicates independent determination of the $\left\langle K_{s}^{o}\right\rangle /\left\langle h^{-}\right\rangle$ratio based on the $K^{+}$and $K^{-}$ measurements and isospin symmetry. The $\left\langle h^{-}\right\rangle$axis represents centrality: central collisions are those of large $\left\langle h^{-}\right\rangle$, peripherals have small $\left\langle h^{-}\right\rangle$. This normalization to the average multiplicity of negative hadrons is very informative: for "normal" events, strange and negative pion multiplicity will grow in the same way with centrality. This would correspond to a constant ratio $\left\langle n_{s}\right\rangle /\left\langle h^{-}\right\rangle$, whereas an increase 
in $\left\langle n_{s}\right\rangle /\left\langle h^{-}\right\rangle$might indicate "something new."

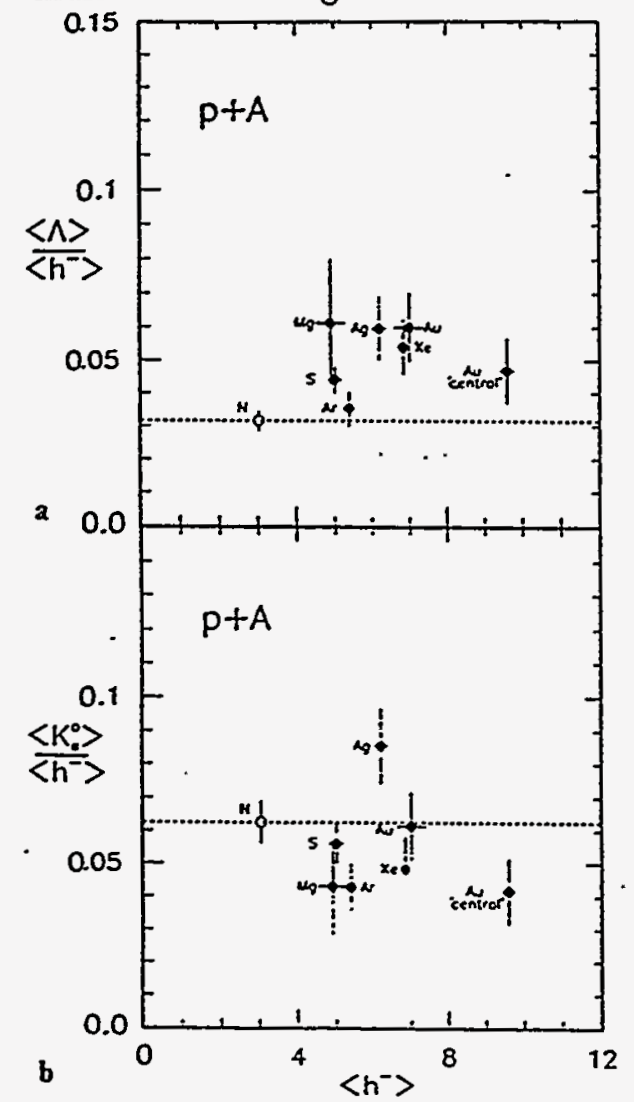

Fig.1a,b. The ratios of the average $\Lambda$ (a) and $K_{s}^{o}$ (b) multiplicities to the average multiplicity of negative hadrons, $\left\langle h^{-}\right\rangle$, in proton-nucleon (open circles) and proton-nucleus (full circles) in-. teractions as a function of $\left\langle h^{-}\right\rangle$in various reactions at $200 \mathrm{GeV}$.

The data on average multiplicities in $\mathrm{p}+\mathrm{A}$ collision (Fig.1a,b) was collected in three different experiments: NA5[5], E565/570[6], and NA35[7]. The data for S+S collisions was obtained by the NA35 experiment [7]. All numbers (Fig.1 and 2) represent full phase space measurements. Comparing figures 1 and 2 we see essential differences. In $\mathrm{p}+\mathrm{A}$ collisions the $\langle\Lambda\rangle /\left\langle h^{-}\right\rangle$ratio is systematically higher whereas the $\left\langle K_{s}^{o}\right\rangle /\left\langle h^{-}\right\rangle$ratio is lower than corresponding ratios obtained for $\mathrm{N}+\mathrm{N}$ interactions (dashed line) [8]. The large statistical errors prevent conclusion on systematic dependence of these ratios on $\left\langle h^{-}\right\rangle$. In the case of $S+S$ interactions, both ratios are significantly higher than for nucleon-nucleon collisions. The similar trend was obtained from VENUS model calculations[9], by introducing new mechanisms of double strings (which increases $\Lambda$ yields) and rescattering (which increases $\Lambda$ and 
$\bar{\Lambda}$ yields).

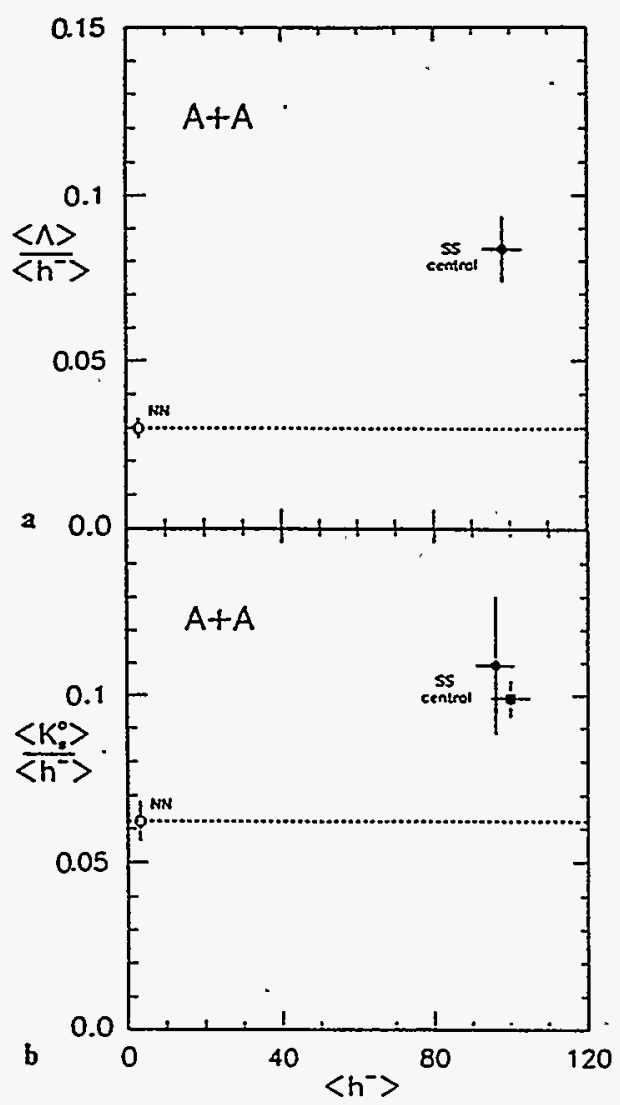

Fig.2a,b. The ratios of the average $\Lambda$ (a) and $K_{s}^{\circ}$ (b) multiplicities to the average multiplicity of negative hadrons, $\left\langle h^{-}\right\rangle$in nucleon-nucleon (open circle) and central $S+S$ (full circles) as a function of $\left\langle h^{-}\right\rangle$at $200 \mathrm{GeV}$. For description of the black square in Fig. 2b, see text.

Since in $\mathrm{p}+\mathrm{A}$ and $\mathrm{A}+\mathrm{A}$ collisions the experimental information on strange and non-strange particle production is usually limited to the yields of negatively charged hadrons, $\Lambda$ 's and $K_{s}^{o}$ 's, it is not practical to apply the strangeness suppression factor $\lambda_{s}$ [10] to characterize strangeness production, because it requires counting of all valence quarks of hadrons at the stage of thermal freeze-out.

Thus, in order to study the total production of strangeness in these reactions in a model independent way using all available experimental information, the following variable was proposed [11]:

$$
E_{s}=\frac{\langle\Lambda\rangle+4\left\langle K_{s}^{\circ}\right\rangle}{3\left\langle\pi^{-}\right\rangle}
$$


where the $\langle\Lambda\rangle,\left\langle K_{s}^{\circ}\right\rangle$ and $\left\langle\pi^{-}\right\rangle$are the mean multiplicities of $\Lambda, K_{s}^{\circ}$, and $\pi^{-}$, respectively. For an isospin zero system this ratio is equivalent to:

$$
\frac{\langle\Lambda\rangle+\langle K+\bar{K}\rangle}{\langle\pi\rangle}
$$

where $\langle K+\bar{K}\rangle$ is the mean multiplicity of neutral and charged kaons and $\langle\pi\rangle$ is the mean multiplicity of pions produced in the strong interaction process.

\begin{tabular}{|c|c|c|c|}
\hline Reaction & $\left\langle h^{-}\right\rangle$ & $\left\langle\pi^{-}\right\rangle$ & $E_{S}$ \\
\hline $\mathrm{N}+\mathrm{N}$ & $3.22 \pm 0.06$ & $3.06 \pm 0.08$ & $0.100 \pm 0.010$ \\
\hline $\begin{array}{c}\mathrm{p}+\mathrm{Mg} \\
\mathrm{p}+\mathrm{Ar} \\
\mathrm{p}+\mathrm{S}\left(h^{+/-}>\mathrm{S}\right)\end{array}$ & $\begin{array}{c}5.39 \pm 0.17 \\
5.7 \pm 0.2\end{array}$ & $\begin{array}{c}5.26 \pm 0.13 \\
\text { averaged }\end{array}$ & $\begin{array}{c}0.086 \pm 0.008 \\
\text { averaged }\end{array}$ \\
\hline $\begin{array}{c}\mathrm{p}+\mathrm{Ag} \\
\mathrm{p}+\mathrm{Xe} \\
\mathrm{p}+\mathrm{Au}\end{array}$ & $\begin{array}{c}6.2 \pm 0.2 \\
7.84 \pm 0.13 \\
7.0 \pm 0.4\end{array}$ & $\begin{array}{c}6.4 \pm 0.11 \\
\text { averaged }\end{array}$ & $\begin{array}{c}0.108 \pm 0.009 \\
\text { averaged }\end{array}$ \\
\hline $\begin{array}{c}\mathrm{p}+\mathrm{Au} \\
\mathrm{central}\end{array}$ & $9.6 \pm 0.2$ & $9.3 \pm 0.2$ & $0.073 \pm 0.015$ \\
\hline${ }^{32} \mathrm{~S}+\mathrm{S}$ & $98 \pm 3$ & $91 \pm 3$ & $0.176 \pm 0.012$ \\
\hline${ }^{32} \mathrm{~S}+\mathrm{Ag}$ & $170 \pm 8$ & $158 \pm 8$ & $0.163 \pm 0.017$ \\
\hline
\end{tabular}

Table 1. The mean multiplicities of negatively charged hadrons, $\pi^{-}$mesons and $E_{s}$ ratio (see text) for nuclear collisions at $200 \mathrm{GeV}$ per nucleon. In order to reduce the large fluctuations present in p-A data - the pion multiplicities and $E_{s}$ values from reactions with similar $\left\langle h^{-}\right\rangle$values were averaged.

The values of $E_{s}$ for nucleon-nucleon [8], proton-nucleon $[5,6,7]$ and central $\mathrm{S}+\mathrm{S}$ and $\mathrm{S}+\mathrm{Ag}[11]$ collisions at $200 \mathrm{GeV}$ per nucleon are given in Table 1 together with the mean multiplicities of negatively charged hadrons and $\pi^{-}$mesons. The $E_{s}$ values for proton-nucleus interactions are approximately equal to the value for nucleon-nucleon interactions. They seem to be independent of the target mass number. The $E_{s}$ values for central $\mathrm{S}+\mathrm{S}$ and $\mathrm{S}+\mathrm{Ag}$ collision are similar and about two times higher than the corresponding $N+N$ and $p+A$ values. This observed increase of the ratio of strange to non-strange hadron yields in comparison to the corresponding ratio for nucleon-nucleon collisions is, according to our adapted definition, the strangeness enhancement. Let us note that the strangeness enhancement is about the same in $\mathrm{S}+\mathrm{S}$ as in $\mathrm{S}+\mathrm{Ag}$, which is in contradiction with the secondary 
processes, occurring in the high energy density regions of the intersection, scenario. In this case one expects higher strangeness enhancement in $\mathrm{S}+\mathrm{Ag}$ than in $\mathrm{S}+\mathrm{S}$, where low density "corona" surface interactions play an important role.

However, one has to be very careful to rely on total integrated yields of particles alone and has to look for confirmation of the findings in particle spectra. It might happen that total integrated yields look similar in the data and the model, whereas separately $\Lambda, \bar{\Lambda}$ and $K_{s}^{o}$ experimental yields can be described simultaneously [9] by theoretical calculations.

\section{Spectra}

Fig. 3 and 4 show the rapidity distribution of $\Lambda$ hyperons produced in central S+S and $\mathrm{S}+\mathrm{Ag}$ collisions observed by the NA35 experiment.

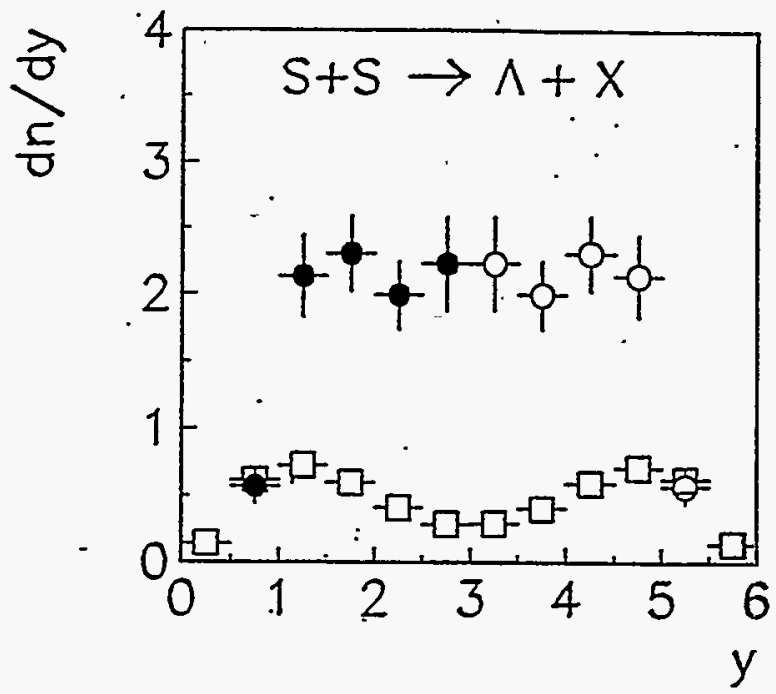

Fig.3. The rapidity distribution of $\Lambda$ hyperons produced in central S+S collisions at $200 \mathrm{GeV} / \mathrm{c}$ per nucleon (circles) and the scaled (see text) rapidity distribution of $\Lambda$ hyperons produced in $\mathrm{N}+\mathrm{N}$ interaction at $200 \mathrm{GeV} / \mathrm{c}$ (squares). The open circles indicate the data reflected with respect to $y_{\mathrm{cm}}$.

The rapidity distributions of $K_{s}^{o}$ mesons are shown in Fig. 5 and 6 , respectively. These distributions are compared with the corresponding distributions obtained for $N+N$ interactions (the reference data for $S+S$ collisions) [8] and $p+S$ interactions (the reference data for $\mathrm{S}+\mathrm{Ag}$ collisions) [11] at $200 \mathrm{GeV} / \mathrm{c}$. The spectra for $N+N$ interactions are multiplied by the ratio $\left\langle\pi^{-}\right\rangle_{S S} /\left\langle\pi^{-}\right\rangle_{p S}$ where $\left\langle\pi^{-}\right\rangle$ is the mean multiplicity of $\pi^{-}$mesons produced in the collisions indicated by the 
index.

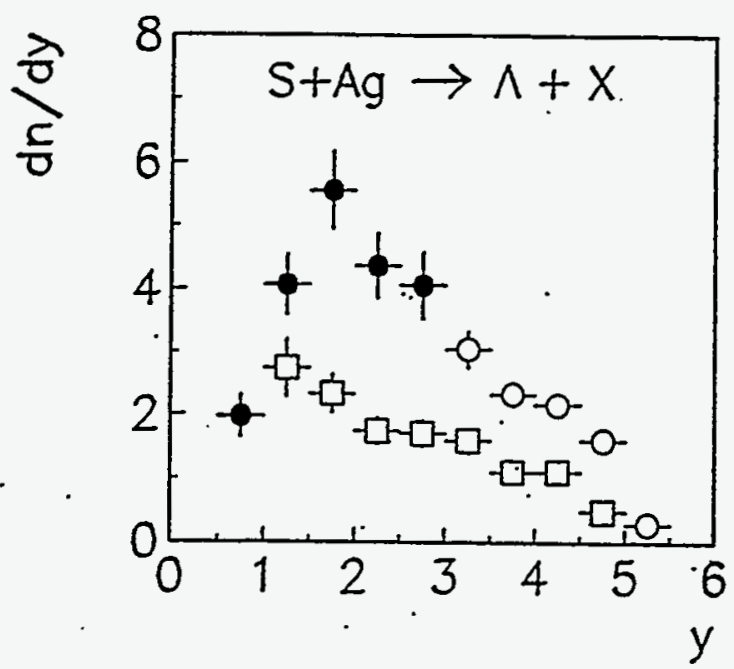

Fig.4. The rapidity distribution of $\Lambda$ hyperons produced in central S+Ag collisions at $200 \mathrm{GeV} / \mathrm{c}$ per nucleon (circles) and the scaled (see text) rapidity distribution of $\Lambda$ hyperons produced in $\mathrm{p}+\mathrm{S}$ interactions at $200 \mathrm{GeV} / \mathrm{c}$ (squares). The open circles indicate the data obtained by an interpolation procedure.

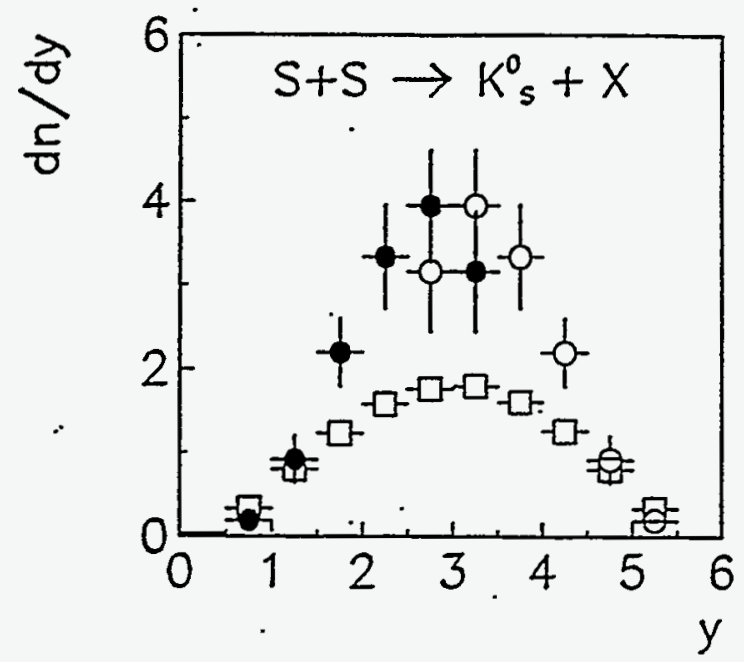

Fig.5. The rapidity distribution of $K_{s}^{o}$ mesons produced in central S+S collisions at $200 \mathrm{GeV} / \mathrm{c}$ per nucleon (circles) and the scaled (see text) rapidity distribution of $K_{s}^{\circ}$ mesons produced in $\mathrm{N}+\mathrm{N}$ interactions at $200 \mathrm{GeV} / \mathrm{c}$ (squares). The open circles indicate the data reflected with respect to $y_{c m}$. 


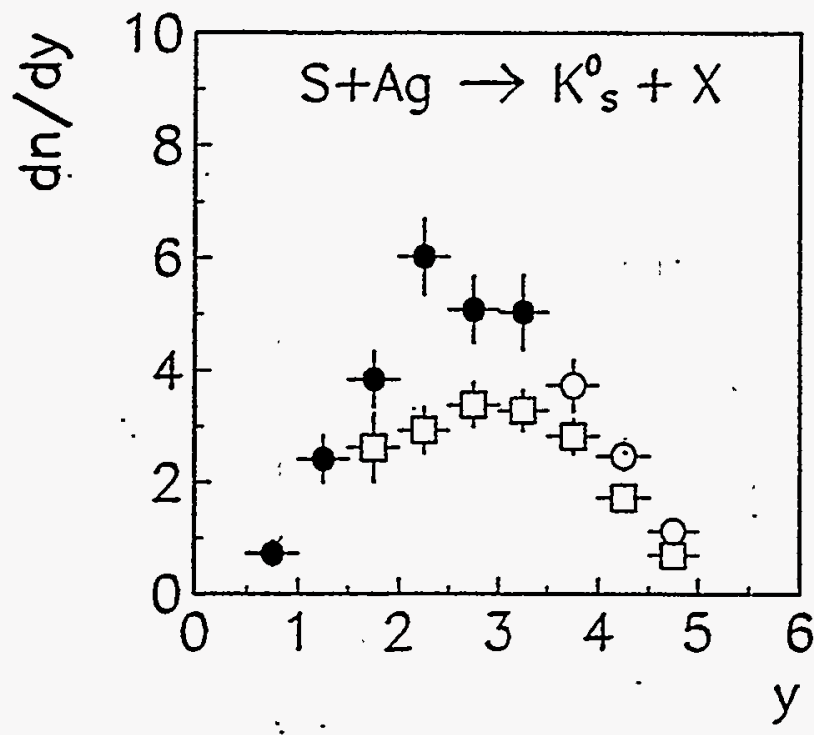

Fig.6. The rapidity distribution of $K_{s}^{o}$ mesons produced in central S+Ag collisions at $200 \mathrm{GeV} / \mathrm{c}$ per nucleon (circles) and the scaled (see text) rapidity distribution of $K_{s}^{0}$ mesons produced in $\mathrm{p}+\mathrm{S}$ interactions at $200 \mathrm{GeV} / \mathrm{c}$ (squares). The open circles indicate the data obtained by an interpolation procedure.

The observed large difference (factor 2-3) between the strange particle yields in central $\mathrm{S}+A_{t}$ collisions and the scaled yields in the reference interactions $(\mathrm{N}+\mathrm{N}$ and $p+S$ ) illustrates the enhanced production of strangeness (relative to $\pi^{-}$mesons) in central sulphur-nucleus collisions previously discussed using variable $E_{s}$ (Table 1).

\section{First look on strangeness production in $\mathrm{Pb}$ on $\mathrm{Pb}$ collisions}

The very first (and very preliminary) results from the NA49 (see paper by S. Margetis in these proceedings) and NA44 (see paper by M. Murray in these proceedings) experiments with a $\mathrm{Pb}$ beam on a $\mathrm{Pb}$ target at the CERN SPS with total energy of $33 \mathrm{TeV}$ show that kaons ( $K_{s}^{o}$ in the case of NA49 and $K^{+}, K^{-}$in the case of NA44) production in $\mathrm{Pb}$ on $\mathrm{Pb}$ is very similar to those observed in $\mathrm{S}+A_{t}$. It is a very intriguing result. We have already eliminated the scenario of rescatttering as a possible source of observed strangeness enhancement based on the fact that strangeness production in $\mathrm{S}+\mathrm{S}$ and $\mathrm{S}+\mathrm{Ag}$ was very similar. Now, we see that this very efficient mechanism of strangeness production which we observe in nucleusnucleus collisions at CERN energies does not depend on the volume of the system and/or on the degree of equilibration. These seem to be counter intuitive and need confirmation and clarification in the future. Figure 7 shows $\left\langle K_{s}^{0}\right\rangle /\left\langle h^{-}\right\rangle$as a function of averages negative hadrons multiplicity in $\mathrm{S}+\mathrm{S}, \mathrm{S}+\mathrm{Ag}$ and $\mathrm{Pb}+\mathrm{Pb}$. The 
dashed line represents $\mathrm{N}+\mathrm{N}$ data. For comparison relevant points for $\mathrm{p}+\mathrm{S}$ and $\mathrm{p}+\mathrm{p}$ are added.

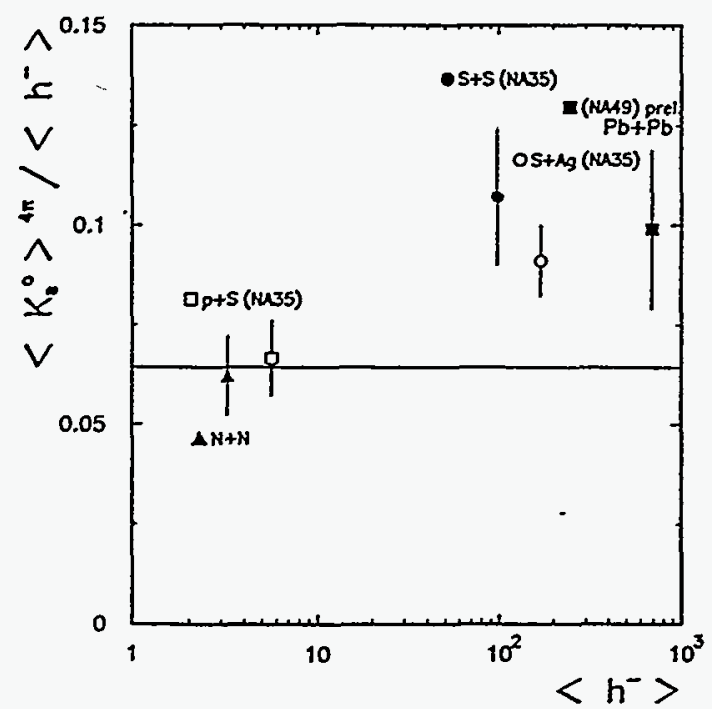

Fig.7. $\left\langle K_{s}^{o}\right\rangle /\left\langle h^{-}\right\rangle$as a function $\left\langle h^{-}\right\rangle$. Points represent $S+S$, $\mathrm{S}+\mathrm{Ag}$ and $\mathrm{Pb}+\mathrm{Pb}$. For comparison $\mathrm{p}+\mathrm{S}$ (open square) and $\mathrm{N}+\mathrm{N}$ (full triangle) points are presented. Solid line represents $N+N$ level.

We see that the $K_{s}^{o}$ production with respect to the average negative hadron multiplicity is the same for all three systems, whereas the $\left\langle h^{-}\right\rangle$changes by factor $\sim 7$. Natural confirmation of this result should come from analysis of hyperon production in $\mathrm{Pb}$ on $\mathrm{Pb}$ collisions. Such an analysis was undertaken by NA49 collaboration. The preliminary invariant mass spectra for $\Lambda$ and $\bar{\Lambda}$ were already presented at QM'96 conference [12], however data was not fully corrected yet which prevented meaningful comparisons with other systems. For the moment the question remains open.

Another intriguing observation of strangeness production in $\mathrm{Pb}$ on $\mathrm{Pb}$ collisions was reported by WA97 (see the paper by H. Helstrup in these proceedings). Namely, it appears that cascades $(\mathrm{S}=2)$ production in $\mathrm{Pb}$ on $\mathrm{Pb}$ interactions seems to be relatively less abundant than production of the omegas $(S=3)$ when normalizing to production in $\mathrm{p}+\mathrm{Pb}$ collisions at the same energy. It is another example of the counter-intuitive observation, because there is no presently known strangeness production mechanism which would favor a process leading to the formation of $\mathrm{S}=3$ particles over those of $\mathrm{S}=2$. 


\section{Looking towards the future...}

All CERN-SPS "heavy" ion experiments involved in the strangeness production studies report strong enhancement, relative to proton-proton and proton-nucleus collisions, of all strange particle yields, which are found to increase with the centrality of the collision $[7,13,14,15,16]$.

While these results have generated much discussion and speculation, they are not sufficiently consistent among themselves to permit definitive conclusions about possible phase transition and/or plasma formation.

The preliminary nature of the data has not discouraged theorists from inserting these experimental results into a variety of models. The most tantalizing theoretical results of such exercises are thermal parameters, vanishing strangeness chemical potential $\mu_{s}$, and values of the strangeness saturation factor $\gamma_{s}$ as high as $60-90 \%$, as extracted from the data using a thermal model generalized to allow for non-equilibrium strange particle abundance[17]. The latter results suggest the presence of an unusually efficient strangeness production mechanism in the heavy ion collisions, as expected if a quark-gluon plasma were formed. At the same time there is no consistent microscopic model capable of describing in full detail the strange particle production seen at CERN. Special features of strangeness production must be inserted "by hand" into particle cascade models to replicate the observed strange particle production enhancement.

Because the enhanced yield of strange particles observed at CERN energies lacks any explanation within the existing hadronic scenarios, strangeness has become the most promising candidate signature of the hypothetical and elusive quarkgluon plasma. It is clear that measurements of both heavy quark flavors, strangeness and charm, in the same collisions with the same apparatus and together with event by event characterization of the collisions should allow more definitive data which should prove (or falsify) the models.

However, for the case of open charm, the question of the production rate at CERN SPS energies remains unanswered. Within a very conservative "ordinary" hadronic scenario (extrapolated from p-p interactions), one would expect one $D$ meson in four $\mathrm{Pb}+\mathrm{Pb}$ central collisions at $200 \mathrm{GeV} / \mathrm{c}$ [18]. However, the strangeness enhancement reported by the CERN SPS experiments and the entropy enhancement reported lately by NA49 [19] suggest a significant departure from this "baseline" hadronic scenario. They suggest that some state of equilibrium, and perhaps even deconfined matter, may be created at energies below that of the CERN SPS. Within such a scenario, the abundant production of charmed mesons may already be taking place, undetected for the time being, at SPS energies because no present CERN heavy ion experiment can detect $D$ mesons. With the completion of the light/medium ion program (O and $\mathrm{S}$ beams) at CERN, the NA36 experiment was terminated. Out of two remaining strangeness experiments, NA49 and WA85, which carry the program to the lead beam era, only NA49 (former NA35) will cover a significant region of the phase space which will allow the study of the correlation 
of strangeness production with the global observables of the event. In the absence of definite signatures for the QGP, it is imperative that such correlations are used to identify special events and possible signatures.

\subsection{NA49 Vertex Silicon Detector (VSD) for strangeness and charm study}

The detection of multi-strange and charmed particles requires precise measurement of secondary vertices close to the target interaction point.

Presently, NA49 collaboration is planning to upgrade the experiment layout by addition of a vertex silicon detector which will have excellent spatial resolution, will be positioned closed to the interaction point and, when combined with the momentum determination and particle identification capabilities provided by the main tracking detectors of the NA49 experiment (four large TPC's), will enable isolation of secondary vertices and determination of particle decay kinematics.

NA49-VSD will use silicon drift detector technology based on p-type silicon substrate developed at LBL [20].

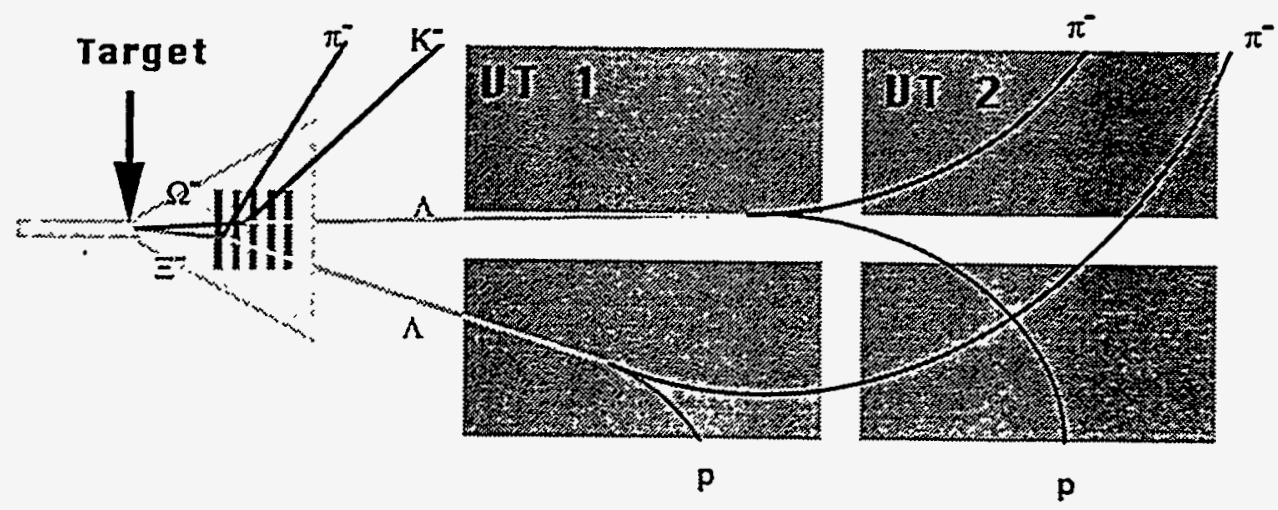

Fig.8. The scematic picture of the NA49 experiment with the VSD. The VSD detector is positioned $8 \mathrm{~cm}$ downstream from the target and in front of two vertex TPC's (VT1 and VT2). Schematics of $\Xi$ and $\Omega$ decays are shown.

Detailed simulations to study the detection of short lived particles in a high multiplicity environment have been carried out to guide a preliminary design of the NA49-VSD.

These simulations have emphasized the detection of $D$ mesons because of their extremely short decay lengths: $c \tau\left(D^{\circ}\right)=126 \mu, c \tau\left(D^{ \pm}\right)=320 \mu$, as compared to $c \tau(\Omega)=2.46 \mathrm{~cm}$ and $c \tau\left(\Xi^{ \pm}\right)=4.91 \mathrm{~cm}$. The experimental requirements for the detection and reconstruction of $D$ mesons are therefore much more demanding and 
complex than those for multistrange hyperons. The simulations indicate that the algorithm, once developed for $D$ mesons, can successfully be relaxed to accommodate longer-lived species $\left(K_{s}^{o}, \Lambda, \Xi, \Omega\right)$ with decay lengths of a few centimeters. The demonstrated ability of other CERN experiments (e.g. NA36, WA85) to reconstruct multistrange hyperons supports this conclusion.

In these simulations the NA49-VSD was assumed to consist of five layers of p-type silicon drift detectors (p-SiDD) spaced $4 \mathrm{~cm}$ apart (Fig.8), with the first layer positioned $8 \mathrm{~cm}$ from the target.

The p-SiDD was assumed to have a space-point accuracy of $20 \mu$. These assumptions reflect measured values. In the detection and reconstruction of multistrange hyperons and $D$ mesons, combined information from the VSD and two vertex TPC's (VT1 and/or VT2) is used.

The $D^{\circ}$ and $D^{ \pm}$charmed mesons from $\sqrt{S}=20 \mathrm{GeV}$ pp events produced by the Pythia event generator were used to evaluate the acceptance and feasibility of detection of open charm mesons in NA49. The background was provided by the $160 \mathrm{GeV} / \mathrm{A} \mathrm{Pb}+\mathrm{Pb}$ events obtained from the VENUS Monte Carlo. Both signal and background events were passed through the NA49 Monte Carlo simulation program (based on GEANT) which included the VSD. Only the charged decay modes $D^{o} \rightarrow K^{-}+\pi^{+}, D^{\circ} \rightarrow K^{+}+\pi^{-}(4.0 \%)$ and $D^{+} \rightarrow K^{+}+\pi^{+}+\pi^{-}$, $D^{-} \rightarrow K^{-}+\pi^{+}+\pi^{-}(9.1 \%)$ were used because of their relatively large branching ratio (see Table 2), the high efficiency of identifying their decay products and their relatively low combinatorial background.

\begin{tabular}{|c|l|c|c|}
\hline Mass (GeV) & Decay Mode & BR (\%) & $\mathrm{c} \tau(\mu \mathrm{m})$ \\
\hline 1.8693 & $\mathrm{D}^{+} \rightarrow \mathrm{K}^{-} \pi^{+} \pi^{+}$ & 9.3 & 320 \\
\hline 1.8645 & $\mathrm{D}^{0} \rightarrow \mathrm{K}^{-} \pi^{+}$ & 4.0 & 126 \\
\hline
\end{tabular}

Table 2. Interesting $D$ meson decay modes for NA49. Charge conjugate modes are implied.

The Lorentz boost at CERN SPS energies $(\gamma \sim 10-20)$ increases the $D$ meson decay length from $100\left(D^{\circ}\right)-300\left(D^{ \pm}\right) \mu$ to more than a centimeter, greatly simplifying the task of separating the decay vertices.

To directly test the feasibility of open charm detection in the presence of a large combinatorial background, we created a Monte Carlo sample of $450 \mathrm{~Pb}$ on $\mathrm{Pb}$ VENUS events with 4 charged $D$ mesons added to each event, thus combining an enriched $D$ signal with normal event background. These events were processed with the NA49 GEANT and pattern recognition programs. Figure 9 shows the invariant mass of reconstructed $D^{ \pm}$mesons found in the 450 event sample.

These results can be extrapolated to the total number of $10^{6}$ events (which corresponds to the two week running time) with $1 \mathrm{D}$ meson per 4 central $\mathrm{Pb}$ on $\mathrm{Pb}$ 
events. With the $\sim 10 \%$ efficiency of our present pattern recognition one expects to have $(7 \pm 1) \sigma D^{ \pm}$signal over the combinatorial background. The work on improving reconstruction efficiency and reducing the background is in progress.

However, even the present algorithm (and assuming no $D$ enhancement at CERN SPS energies), can be expected to reconstruct few hundreds of $D$ mesons in all channels in $10^{6}$ central $\mathrm{Pb}+\mathrm{Pb}$ events. This is a comfortable number that should permit the extraction of statistically valid $y$ and $p_{t}$ distributions.

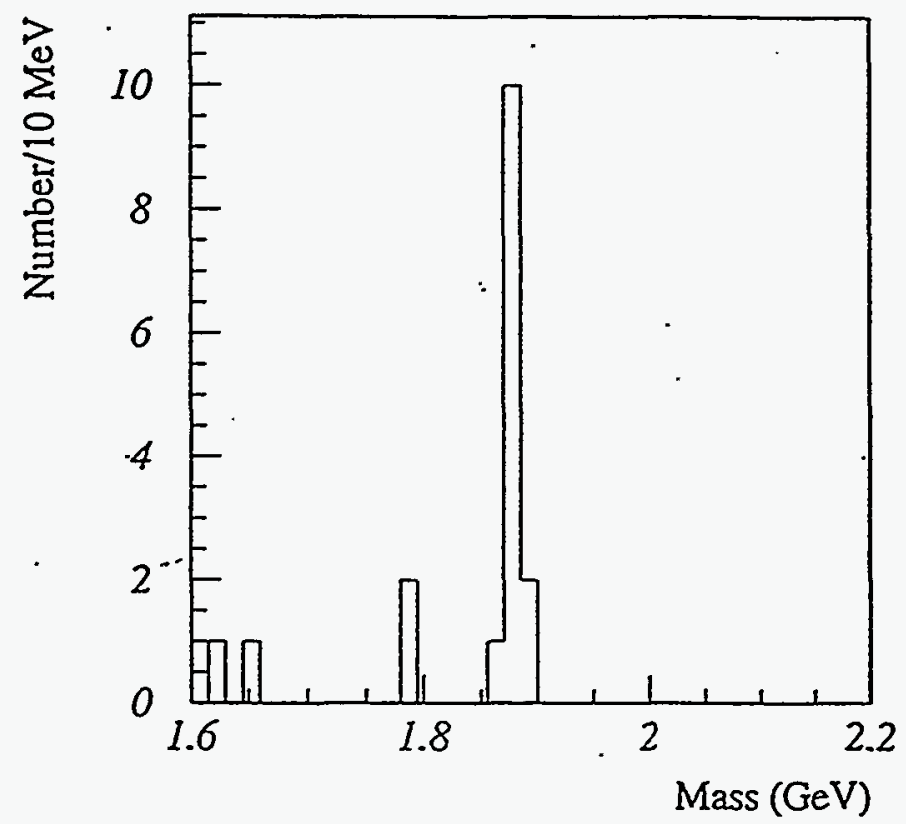

Fig.9. Invariant mass spectrum od $D$ meson candidate vertices found in 450 VENUS events.

\section{Instead of conclusions ...}

It is too early for conclusions. The strangeness enhancement by about factor of 2 in sulphur-nucleus collisions at CERN SPS energies is not consistently reproduced by any calculation based on primary and secondary hadronic and string interactions. The data from $\mathrm{Pb}$ on $\mathrm{Pb}$ collisions are still very preliminary and the analysis is in an early stage. However, first results seem to be consistent with observations from experiments with sulphur beams and suggest that this unknown, new, and very efficient mechanism, responsible for strange particle production in heavy ion collisions is independent of the volume of the interacting system and/or the degree of equilibration. This is an astonishing result and clearly needs confirmation in the next stages of the analysis with larger statistics. 
A lot of data were gathered during the first $\mathrm{Pb}$ run at the CERN SPS and only a small part has, so far, been analyzed. A number of new data taking runs are planned for the coming years (about one per year) and much more data will be collected. The large systems are clearly more amenable to a thermal description and they are also expected to show signals more clearly, but they are far more complex (and CPU "intensive") than anything we have analyzed before. Therefore, it might be a quite some time before we will be able to draw definite conclusions on strangeness and other issues of QGP formation ...

\section{References}

1. M.Gazdzicki and NA35 Coll. Nucl.Phys.A498(1989)375.

2. P.Koch, B.Muller, J.Rafelski, Phys.Rep.142(1986)165.

3. J.Kogut, D.Sinclair, Phys.Rev.Lett.60(1988)1250.

4. H.Bialkowska et al., Z.Phys.C55(1992) 491 .

5. I.Dorado et al., Z.Phys.C50(1991)31.

6. D.H.Brick et al., Phys.Rev.D45(1992)734.

7. J.Bartke et al., Z.Phys.C48(1990)191, J.Bachler et al., Z.Phys.C5(1991)157, M.Kowalski and NA35 Coll. Nucl.Phys. A544(1992)609.

8. M.Gazdzicki, O.Hansen, Nucl.Phys.528(1991)75.

9. K.Werner, Phys.Rep. 232(1993)87.

10. A.K.Wroblewski, Acta Phys.Pol. B16(1985)379.

11. T.Alber et al., Z.Phys.C64(1994)195.

12. P.Jones and NA49 Coll., QM'96 Conf., Heildelberg, May 1996.

13. see Quark Matter'91 Proceedings, Nucl.Phys.A544(1992).

14. D.Evans and WA85 Coll:, Nucl.Phys.A566(1994).

15. S.Abatzis et al., Phys.Lett.B270, 123 (1991); 259,508(1991).

16. E.Andersen and NA36 Coll., Phys.Lett.B294,127(1992).

17. J.Rafelski, M.Danos, Phys.Rev.C50,(1994)1684.

18. R.Vogt - Private communication.

19. M.Gazdzicki and NA35 Coll., Nucl.Phys.A590(1995)197.

20. G.Odyniec et al., AIP Conf.Proc.340,Tucson,1995,p.90. 